\title{
Current and future needs and research for composite materials NDE
}

\section{K. Elliott Cramer}

K. Elliott Cramer, "Current and future needs and research for composite materials NDE," Proc. SPIE 10596, Behavior and Mechanics of

Multifunctional Materials and Composites XII, 1059603 (22 March 2018); doi: 10.1117/12.2291921

Event: SPIE Smart Structures and Materials + Nondestructive Evaluation and Health Monitoring, 2018, Denver, Colorado, United States 


\title{
Current and Future Needs and Research for Composite Materials NDE \\ K. Elliott Cramer*a \\ ${ }^{a}$ NASA Langley Research Center, 3B E. Taylor St. - MS231, Hampton, VA 23681
}

\begin{abstract}
The use of composite materials continues to increase in the aerospace community due to the potential benefits of reduced weight, increased strength, and manufacturability. The ability to characterize damage in carbon fiber reinforced polymer composite components is required to facilitate damage progression models capable of yielding accurate remaining life predictions. As these composite structures become larger and more complex, nondestructive evaluation (NDE) techniques capable of quantifying and fully characterizing the material state are needed to enable damage progression models capable of yielding accurate remaining life predictions. This paper will present an overview of current NDE research activities for quantitative characterization of aerospace composites as well as a discussion of future directions in NDE research.
\end{abstract}

Keywords: Composites, Nondestructive Evaluation

\section{INTRODUCTION}

In recent years, the aerospace community has increased the use of composites in aeronautic and space vehicles. As demonstrated by the Boeing 787's use of composites ${ }^{[1]}$, NASA's Composite Crew Module ${ }^{[2]}$ and liquid hydrogen (LH2) cryogenic tanks ${ }^{[3]}$, there is a push toward the use of composites for primary structural components. As these composite structures become larger and more complex, nondestructive evaluation (NDE) techniques capable of quantifying and fully characterizing damage are needed. The ability to quantitatively characterize damage in carbon fiber reinforced polymer (CFRP) composite components is required to enable damage progression models capable of yielding accurate remaining life predictions. For example, the depth at which delaminations occur is directly related to how damage growth progresses ${ }^{[4]}$. Therefore, a 'full' characterization of delamination damage needs to go beyond a quantitative measure of the in-plane area (size) of the damage, to include the depth/ply at which the damage occurs. For multilayered delamination damage, a full assessment would ideally include the depth and size of all delaminations, if possible. A 'full' damage characterization for other damage types may require different damage information. Microcracking may be best characterized by a measure of microcrack density correlated to depth through the material, while fiber waviness may require a statistical measure of the affected locations and corresponding ranges in the angle of unintended in-plane or out-of-plane alignment/waviness of fibers (i.e., a 'waviness angle' range) [[],[6]. $^{[}$.

The challenge of acquiring quantitative NDE damage characterization for aerospace composites is compounded not only by the size of the structure and complexity of the damage types occurring in composites, but also by the complex geometries of composite components required for aerospace applications. The research approaches of NASA's Nondestructive Evaluation Sciences Branch (NESB) include investigation of conventional, guided wave and phase sensitive ultrasonic methods, infrared thermography and $\mathrm{x}$-ray computed tomography techniques. The use of simulation tools for optimizing and developing these methods is an active area of research. This paper will focus on an overview of current NDE research activities for quantitative characterization of aerospace composites as well as a discussion of future directions in NDE research.

\section{CURRENT INSPECTION TECHNOLOGIES}

\subsection{Infrared Flash Thermography}

Flash infrared thermography has been used extensively as a large area rapid inspection technology for composite structures. The flash thermography system typically used by NESB is the commercially available Echotherm ${ }^{\circledR}$ system from Thermal Wave Imaging, Inc. The system features a flash hood containing a 1280 x 720 element FLIR A8300sc infrared (IR) camera with two 4800-Joule xenon photographic flash tubes. The hood has dimensions of $36.8 \mathrm{~cm}$ wide by $26.7 \mathrm{~cm}$ deep by $40.6 \mathrm{~cm}$ tall and is configured such that the IR camera views the inspection surface directly. The flash

Behavior and Mechanics of Multifunctional Materials and Composites XII,

edited by Hani E. Naguib, Proc. of SPIE Vol. 10596, 1059603 - @ 2018 SPIE CCC code: $0277-786 \mathrm{X} / 18 / \$ 18 \cdot$ doi: $10.1117 / 12.2291921$ 
produces an energy density of 7.15 joules per square centimeter at the mouth of the hood (based on the final temperature increase of a reference specimen and the nominal thermal properties of that reference). The flash lamp provides a uniform illumination of within $10 \%$ across an area of $24.8 \mathrm{~cm}$ x $32.7 \mathrm{~cm}$ (for a flat reference standard at the mouth of the hood as determined from measurements of the temperature rise of the reference material). The flash creates an initial temperature increase of the inspection surface of less than $10^{\circ} \mathrm{C}$. A photograph of the flash thermography system is shown in figure 1.

The hood is connected to a base station, which houses the system computer and power sources for the various components. Thermographic inspection is accomplished by placing the hood on the section of material to be inspected. The bottom of the hood completely surrounds or sits atop the material, depending on the dimension of the specimen. The flash lamps are triggered either by operator controls on the hood or by the computer. Thermographic images of the specimen are captured by the IR camera for a predetermined amount of time and stored in the computer for further analysis. The camera's noise equivalent temperature difference, cited by the manufacturer, is $0.025^{\circ} \mathrm{C}$. The detector array operates in the 3 to 5 micrometer wavelength range. External optics, consisting of a wide-angle lens ( $25 \mathrm{~mm}$ focal length), using germanium optical elements, are used to increase the system field-of-view to $21.7^{\circ}$ horizontally and $17.5^{\circ}$ vertically.

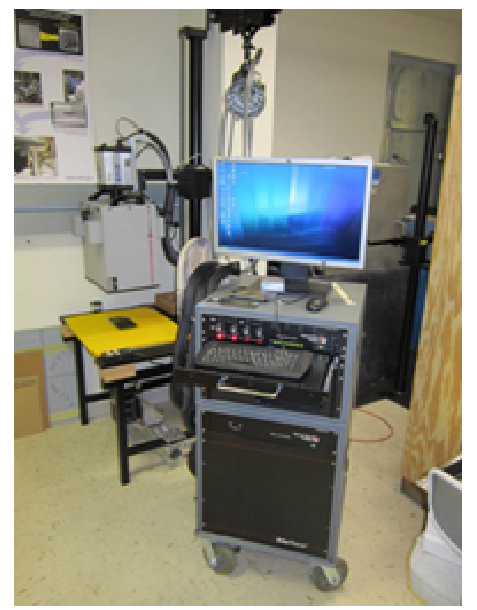

Figure 1. Photograph of the flash thermograph system.

Figure 2 shows an implementation of flash thermography for large area composite inspection. The test specimen is a composite cylinder $10 \mathrm{~m}$ in diameter and $4 \mathrm{~m}$ in height, built as five panels connected with bonded joints. Inspection of the cylinder required approximately 10 hours per side (interior and exterior) and yielded $250 \mathrm{~GB}$ of thermal data. The thermal system is translated over the entire surface of the specimen using a custom scanning rail system that follows the curvature of the cylinder. Data acquisition consists of positioning the camera and hood, triggering the flash heating, collecting data for $15 \mathrm{~s}$ after the heating, storing the data and indexing the scanner to the next inspection location. Total inspection time per location is approximately $45 \mathrm{~s}$. Data analysis was performed using Principal Component Analysis (PCA) with model derived eigenvectors ${ }^{[7],[8]}$. Details of this analysis technique are discussed in section 3.2 of this paper. This approach reduced the processing time per data set $(250 \mathrm{MB})$ to less than $1 \mathrm{~s}$, which is less than the time required to move the camera and hood to the next inspection location. As an example of typical results, figure 3 shows a PCA processed mosaic of the inspections results from a portion of one of the joints on the inner mold line of the specimen. The dark regions of figure 3 appear to be areas of excess resin in the joint, while the light regions (a few of which are indicated by red arrows) are consistent with areas of poor bonding. 


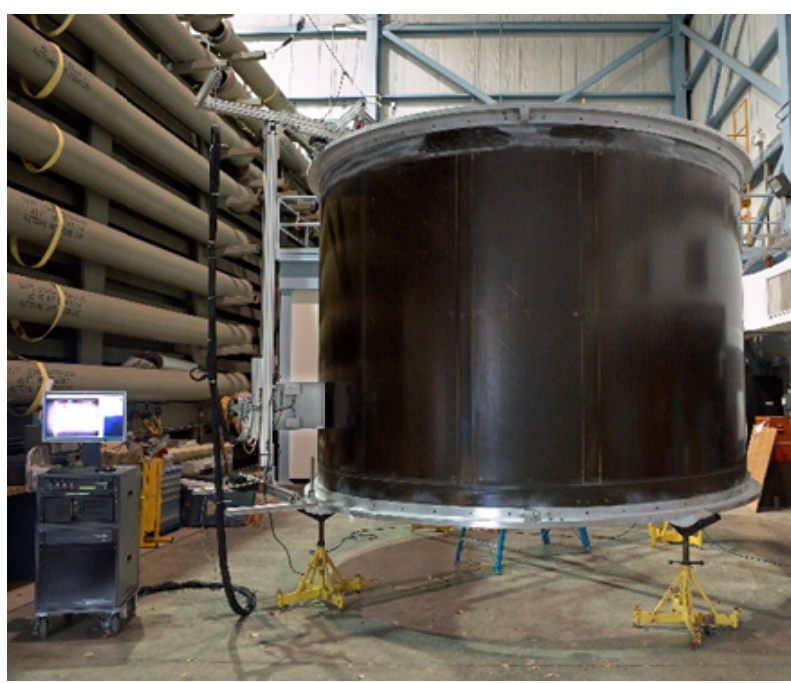

Figure 2. System configured for large area inspection of a composite test article.

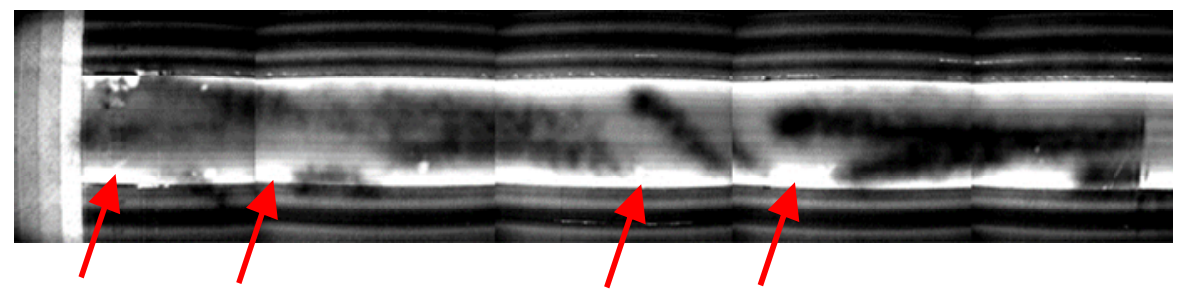

Figure 3. Mosaic images showing PCA processed thermal results for a portion of joint number 2 from the inner mold line.

The red arrows highlight regions along the edge of the joint consistent with areas of poor bonding.

In addition to qualification of manufactured parts, infrared thermography is an ideal candidate technology for in-situ inspection enabling the sustainment of in-service structures. For example, NASA has been using IR thermography for on-orbit inspection of manned flight vehicles for a number of years ${ }^{[9]-[12]}$. Figure 4 shows an IR inspection system configured for use by astronauts during an extravehicular activity (EVA). Figure 5 shows the results of EVA inspections, both from the International Space Station (ISS) and from the Space Shuttle. Figure 5a shows processed results from a space shuttle EVA using the IR inspection system coupled with solar heating for inspection of both damaged reinforced carbon-carbon specimen and an NDE flat-bottom-hole standard. Figure 5b shows processed results from an IR inspection of a damaged ISS radiator. This example shows how the IR imagery temperature data can be analyzed to show that there is heat rejection loss for the area under the peeled-up face sheet ${ }^{[12]}$.

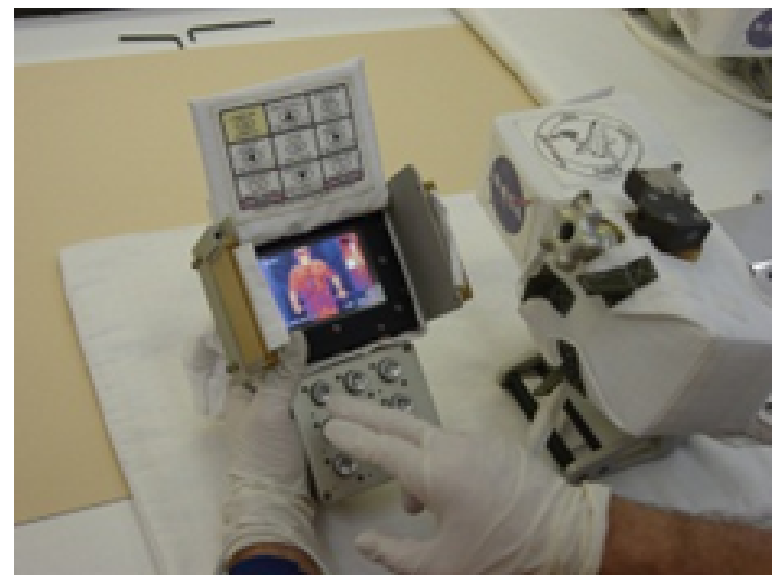

Figure 4. Infrared inspection system designed for on-orbit inspections. 


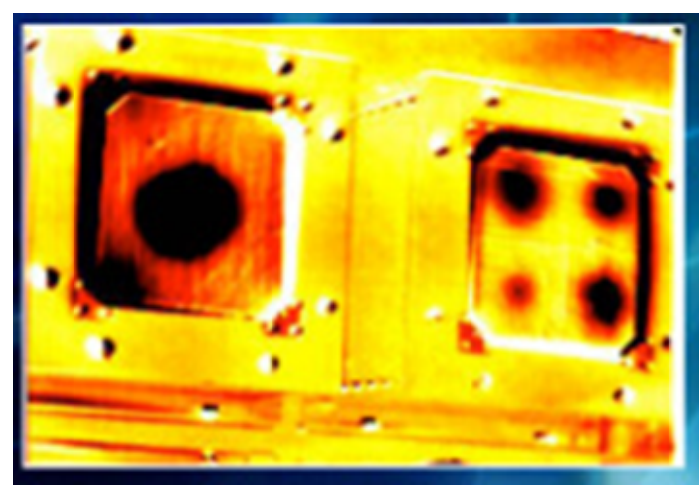

(a)

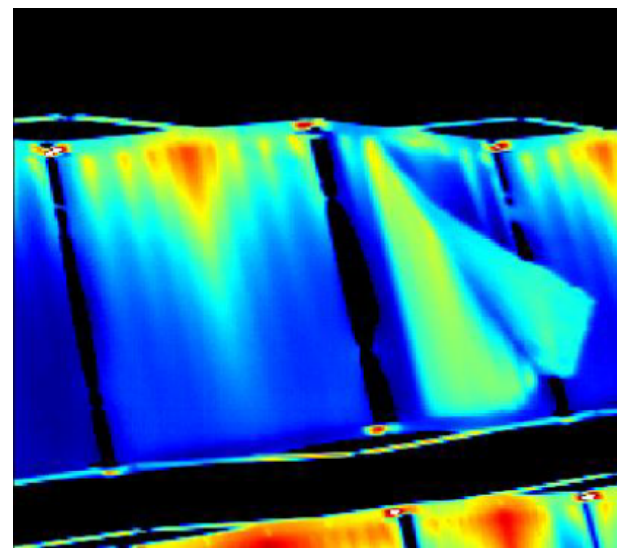

(b)

Figure 5. Infrared inspection results during EVA from (a) Space Shuttle and (b) the ISS.

\subsection{Large Area Ultrasonics}

Ultrasound is a valuable technology for the nondestructive inspection of aerospace structures. In the majority of situations, an ultrasonic probe must be coupled to the structure by physical contact. A fluid medium promotes complete coupling of the acoustic wave into the material. Often, solid coupling devices such as stand-offs or wedges provide time delay and incident angle control of the sound waves into the part. Solid stand-offs and wedges can be shaped to match planar and fixed curvature surfaces. However, ultrasonic coupling problems occur when surfaces deviate from the shape of a solid coupling device. Furthermore, the ultrasonic beam from a contact probe is typically in the near field of the transducer and unfocused. In a given application, the lateral resolution obtained may be inadequate for the required measurement. Therefore, for large area, high speed applications NASA Langley has chosen to implement a captive water column coupling approach ${ }^{[13]}$. This provides coupling that is similar to an ultrasonic water squirter system without requiring a mechanism for delivering and capturing the constant stream of water.

Figure 6a shows a photograph of a single-element transducer with a captive water column. The transducer is sealed into a water-tight probe housing. Water is captured in the probe housing with a flexible membrane selected to produce very little ultrasonic reflection. The membrane is pressed directly against the inspection surface and mechanically scanned. Usually, a mist of water is required to wet the surfaces and promote ultrasonic coupling. A large area, high speed, computer controlled scanning system allows for automated inspections of large specimens. Scanning speeds are a maximum of $2 \mathrm{~m} / \mathrm{s}$ (typical scan speed are $0.3-0.6 \mathrm{~m} / \mathrm{s}$ ) with full waveform capture (16-bit) of the ultrasonic signal every $0.25 \mathrm{~mm}$.

Figure $6 \mathrm{~b}$ shows an example of pulse-echo $\mathrm{C}$-scan results from the inspection of a large composite specimen, approximately $2.4 \mathrm{~m} \times 2.4 \mathrm{~m}$ in size. The test article is a composite sandwich structure with 6-ply graphite/epoxy face sheets and an aluminum honeycomb core. The inset image is a close up, high resolution scan of a delamination between the face sheet and the core. Total acquisition time for the captive water column ultrasonic inspection was approximately 45 minutes per side. 


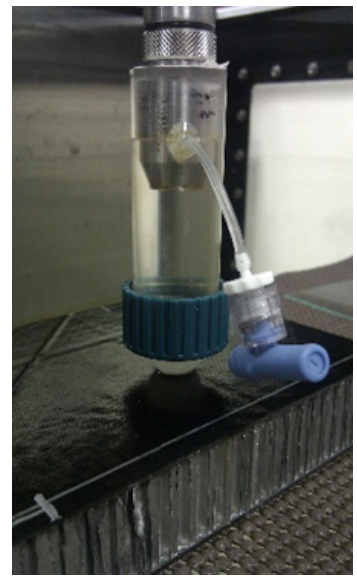

(a)

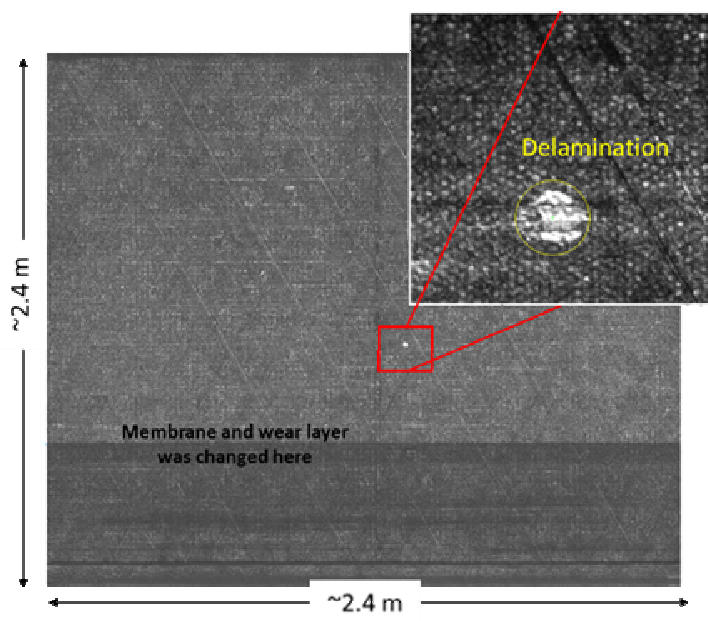

(b)

Figure 6. (a) A photograph of a single-element transducer with a captive water column used for large area, high-speed ultrasound. (b) Ultrasonic C-scan results from the inspection of a large honeycomb sandwich composite specimen with an inset showing a skin-to-core delamination.

\subsection{Other Techniques}

Techniques such as guided wave and polar backscatter ultrasonic methods, eddy current inspection, x-ray computed tomography and backscatter x-ray have all shown promise in the inspection of composite structures for characterization of specific defect types. An example of the application of one of these technologies is shown in figure 7. Oblique incidence pulse-echo ultrasound (also known as polar backscatter) has been used to measure a number of defects found in complex composite structures such as delaminations, transverse matrix cracks and fiber orientation anomalies ${ }^{[13]}$. Figure 7a shows an image of a quasi-isotropic composite specimen with in-plane fiber waviness created near surface by ply shearing. The specimen was scanned using the polar backscatter ultrasonic technique described by Johnston ${ }^{[14]}$ and the scan results, shown in figure $7 \mathrm{~b}$, demonstrate the ability of the technique to characterize the wavy surface plies. Modern phased array instruments have demonstrated sufficiently rapid scan rates to exploit array technology to implement the polar backscatter approach efficiently.

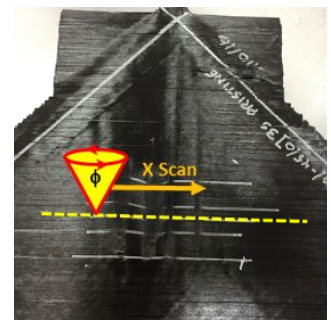

(a)

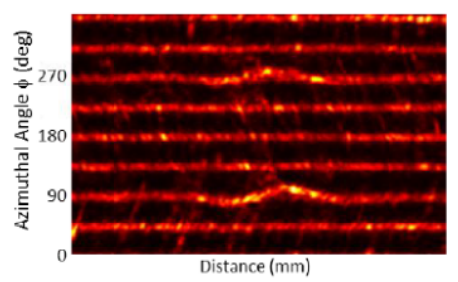

(b)

Figure 7. (a) A photograph of a quasi-isotropic composite specimen fabricated with in-plane fiber waviness. (b) Ultrasonic polar backscatter results (with a $25^{\circ}$ polar angle) from the inspection of the fiber waviness specimen scanned in $\mathrm{x}$ and $\phi$.

\section{FUTURE DIRECTIONS IN NDE}

A critical challenge for future aerospace vehicles is the development of systems that can ensure safety and reliability during increasingly complex missions. These systems will enable the use of lighter weight materials without sacrificing safety. Additionally, assessing and maintaining vehicle integrity with minimal human intervention will be of paramount importance. Accurate characterization of integrity will require in-situ sensor arrays to rapidly interrogate large areas and detect structural anomalies. Deployable, autonomous NDE devices will be needed to perform accurate local assessments of these anomalies. Sensor systems must be capable of detecting precursors of unanticipated global degradation as well as rapidly identifying and locating suddenly occurring mission threatening damage. This will enable early mitigation 
against conditions critical to maintaining vehicle integrity. Game changing technology will be required to produce new classes of sensors and NDE methodologies tailored to specific applications. A combination of fixed global sensor arrays along with mobile, autonomous inspection devices will be required for early detection, quantification, localization, and mitigation of critical conditions.

\subsection{Robotic Implementation of NDE Inspections}

Autonomous inspection capability will be required to ensure reliable structures/systems. To date, a majority of the autonomous inspection technologies that have been developed have been limited to visual inspections and most of these have been applied to the inspection of pipelines ${ }^{[15]}$ or applied to large infrastructure inspections such as in nuclear power plants $^{[16]}$. These systems typically require significant human interaction (either directly or remotely). However, for a fully autonomous inspection, the development technology capable of ensuring that the inspection system is properly configured, registered and coupled to the structure is necessary. Additional needs include systems that are capable of automatically adjusting the required resolution to accurately size and identify flaws, all with minimal intervention of an operator. These systems should also be aware of their location on the structure and capable of mapping the results to a model of the structure under inspection ${ }^{[17]}$. Real time flaw inspection data would link to physics-based models of the vehicle to interrogate vehicle health and plan required damage mitigation strategies. This is critical for long duration missions where an autonomous inspection system would free mission specialist's time to enable them to perform other functions. Additionally, it can be coupled to a robotic system to enable inspections without extravehicular activities, which would support both manned and unmanned vehicles.

Current research in NESB is investigating the use of collaborative robots (cobots). A collaborative robot is a robot intended to physically interact with humans in a shared workspace ${ }^{[18]}$. The use of collaborative robots for NDE is attractive for several reasons. (1) Cobots enable the registration, precision, repeatability and speed that robotics provides while eliminating the need for safety exclusion zones or other safety barriers during inspection. (2) Cobots allows robotic NDE to be performed on a structure or vehicle while other work is taking place. Figure 8 shows two photographs of a cobot performing flash thermography on a composite aircraft fuselage structure. Figure 9 shows the results of a flash thermography inspection of the fuselage. All of the data acquired was registered to its location on the fuselage and processed using PCA as described in sections 2.1 and 3.2 of this paper.
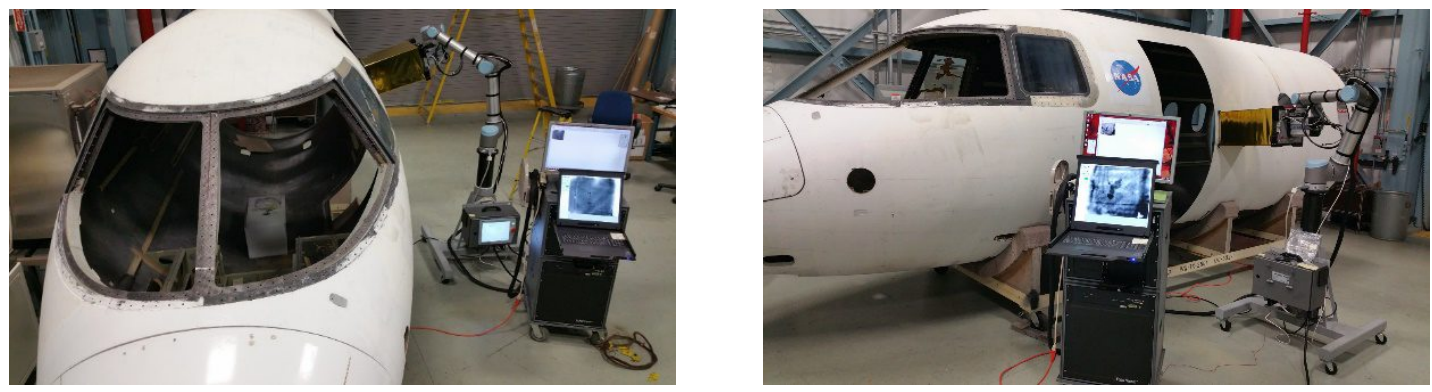

Figure 8. Two photographs of flash infrared thermography inspection performed on a composite aircraft fuselage by a cobot.
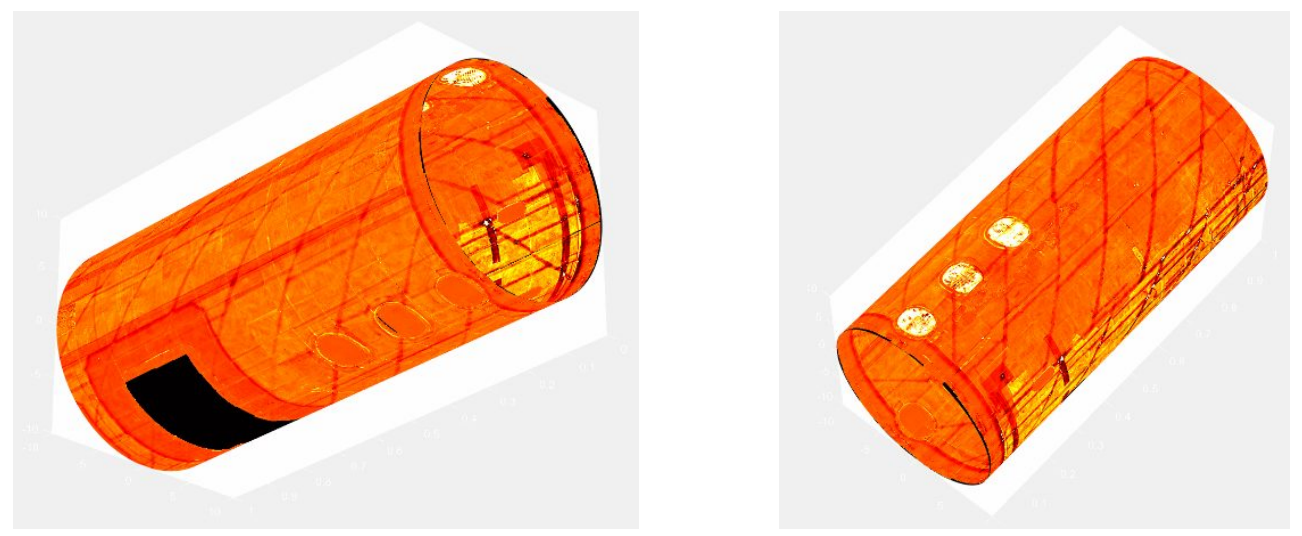

Figure 9. Two 3D views of the processed and registered thermal data from a complete inspection of the composite fuselage. 


\subsection{Computational NDE}

Simulation tools have the potential to create a cost-effective method for developing and optimizing damage characterization techniques for composites. Such tools enable a method for predicting the inspectability of advanced composite components during the design stage. For example, simulation tools could be used to establish confidence in an NDE technique's ability to inspect complex joints, hard to reach locations, characterize complex damage and/or to inspect large areas ${ }^{[19]}$. Additionally, simulation tools can be used to develop new rapid and quantitative methods to analyze NDE data. Therefore, advancing NDE simulation methodologies are an area of active research for NASA's NESB involving the development, implementation and validation of these tools.

To demonstrate the capability of simulating complex damage in a composite material, a 26-ply quasi-isotropic IM7/8552 composite laminate coupon was fabricated at NASA Langley to be used for the purpose of growing enclosed impact-like delamination damage. The composite coupon is $152 \mathrm{~mm}$ by $65 \mathrm{~mm}$ by $3.23 \mathrm{~mm}$. Details of the damage growth setup can be found in a prior paper by Rogge and Leckey ${ }^{[20]}$. The damaged sample was scanned at a resolution of $23.4 \mu \mathrm{m}$ using microfocus x-ray computed tomography (microCT). Figure 10a shows the microCT results scaled such that only the delamination is visible. The interaction of guided ultrasonic waves with the delamination damage defined by the microCT was then modeled using custom developed 3D anisotropic elastodynamic finite integration technique (EFIT) software $^{[21]}$. For straight-forward one-to-one mapping of the damage into the simulation, and to capture all details of the delamination damage, the EFIT simulation used a step size that is equal to the microCT data resolution $(23.4 \mu \mathrm{m})$. Figure 10b shows a 2D representation of a through-thickness slice from the EFIT simulation results at four different time steps and demonstrates that the complex geometry of the damage must be taken into account in order to capture the scattering and mode conversion that occurs as guided waves interact with the delaminated region. Modeling such a complex delamination as a simple circular void would not accurately capture this behavior. Analysis of the simulation results has led to an approach for the detection of hidden multilayer delaminations ${ }^{[22]}$.

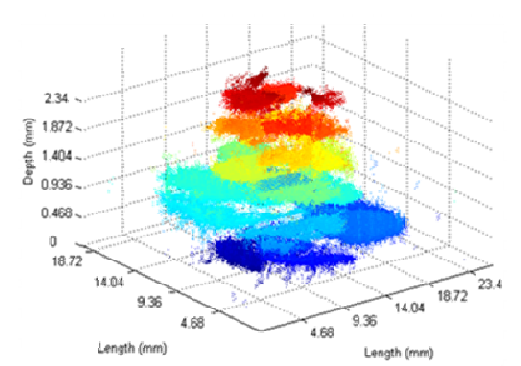

(a)

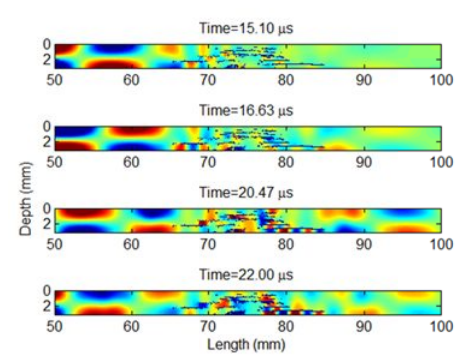

(b)

Figure 10. (a) MicroCT results showing the complex nature of impact damage in a composite specimen and (b) simulation of guided ultrasonic wave in the presence of complex delamination damage.

Finally, consider an example of how simulation tools can be used to develop new rapid and quantitative methods to analyze NDE data. PCA has been used extensively for the reduction of flash IR thermography data. Typical application of PCA to reduce transient thermographic data consists of calculating the principal components of the temporal data through singular value decomposition (SVD) of the experimental data itself. For example, Rajic ${ }^{[23],[24]}$ and Valluzzi ${ }^{[25]}$ both use PCA as a contrast enhancement technique for defect detection. Genest ${ }^{[26]}$ and Vavilov ${ }^{[27]}$ provide comparisons between PCA and various other data reduction techniques for defect sizing. Zalameda ${ }^{[28]}$ discusses PCA's use for temporal compression of the thermal data. PCA was used to analyze thermal "flying-spot" data by Hermosilla-Lara ${ }^{[29]}$ for detection of open cracks in metallic specimens. Finally, Marinett ${ }^{[30]}$ suggests the use of an experimentally derived training set to calculate the principal components.

While this technique is quite effective in reducing thermal data, the singular value decomposition require for PCA can be computationally intense especially with large three-dimensional arrays of thermal data typically produced during an inspection. Additionally, PCA can experience problems when very large defects are present (defects that dominate the field-of-view). The first vector accounts for as much of the variability in the data as possible therefore if defective material dominates the field of view, the first eigenvector may reflect the response of the defect, not the "good" material. If material responses captured by the first eigenvector are considered a nominal material response, then a misclassification of the defect region can result. To increase the processing speed and eliminate issues arising from the 
presence of large defects, an alternative method of PCA is being pursued where a fixed set of eigenvectors, generated from an analytic model of the thermal response of the material under examination, is used to process the thermal data from composite materials. Either a one-dimensional (1D) multilayer analytic model or a 2D finite element model is used and a set of eigenvectors are then numerically generated from this array of responses ${ }^{[7],[8]}$. Figure 3 , discussed earlier, shows the results achieved using this model based analysis approach. In this example, calculation of each PCA image (the mosaic of figure 3 contains 10 PCA images) using the model derived eigenvectors took less than 1 second in Matlab ${ }^{\circledR}$, whereas a full SVD calculation of the eigenvectors and back projection on the same data can take more than 1 minute.

\subsection{In situ Inspection of Composite Manufacturing}

Automated fiber placement (AFP) is the process by which fibers, preimpregnated with resin, are placed layer-by-layer using a robotic system to build up the composite laminate. The composite laminate is then cured, usually in an autoclave. This process for composite part fabrication by AFP can result in a number of potential flaws, the simplest of which are gaps (unintended space between tows), laps (when tows overlap) and twists (when a tow is twisted and a portion of it does not lie flat). These AFP flaws are typically found by time intensive visual inspection. An AFP part might also contain flaws that are much more difficult to detect visually such as insufficient adhesion between plies or tow peel-up in the presence of complex geometry. Finding flaws and correcting them prior to placing another layer or prior to cure saves material and time as correcting flaws post cure is much more complicated. Infrared thermography ${ }^{[31]}$ and microwave imaging ${ }^{[32]}$ are both being investigated for use to characterize these defects during the AFP process

For example, thermography provides a direct measure of the surface temperature of a part. Additionally, thermography provides information about volume under inspection by observing the surface temperature over time in the presence of known heating conditions. The AFP process utilizes a heat source to aid with compaction and adherence of the fibers. By observing and analyzing the time history of the surface after a new course (or layer of fibers) is applied the part quality can be assessed. This type of assessment allows repairs to be made during fabrication, reducing the risk of curing a part of unacceptable quality. Figure 11 shows a photograph of NASA Langley's AFP system with a close-up of the integrated IR camera for in situ inspection during fiber placement. Figure 12 shows some the individual frames captured by the in-situ thermal camera. Figure 12a shows examples of fiber tows that are peeling-up due to the steering angle compared to figure $12 \mathrm{~b}$ that shows tows without the peel-up. The black corresponds to cooler temperatures, the tow peelup appear as dark, cool spots where the fiber tows have separated from the hotter substrate.

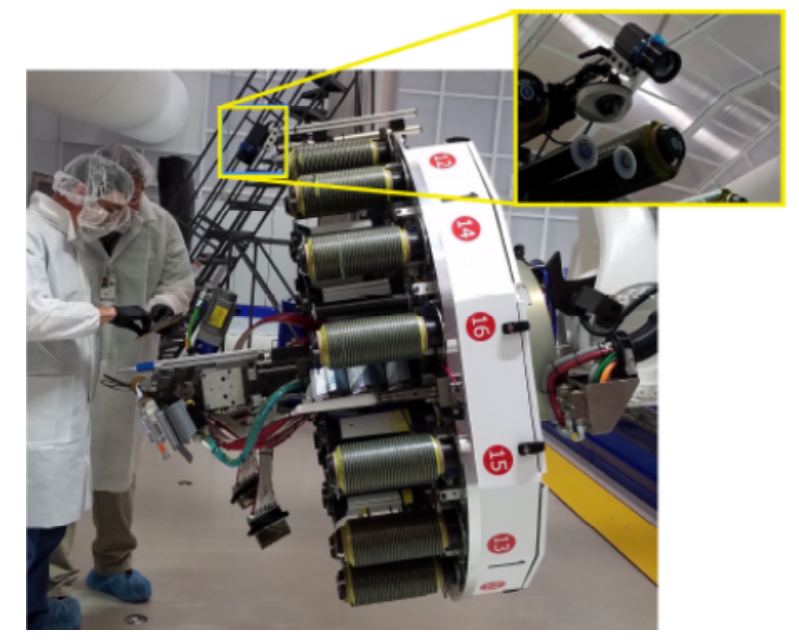

Figure 11. Integrated thermal camera on AFP system at NASA Langley. 


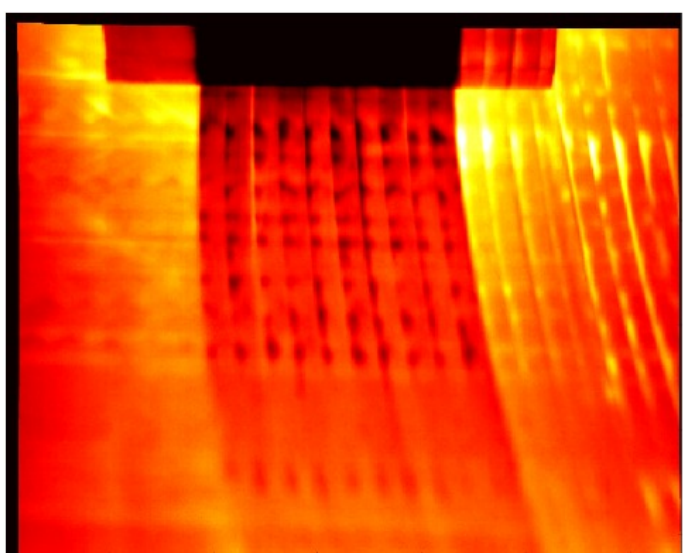

(a)

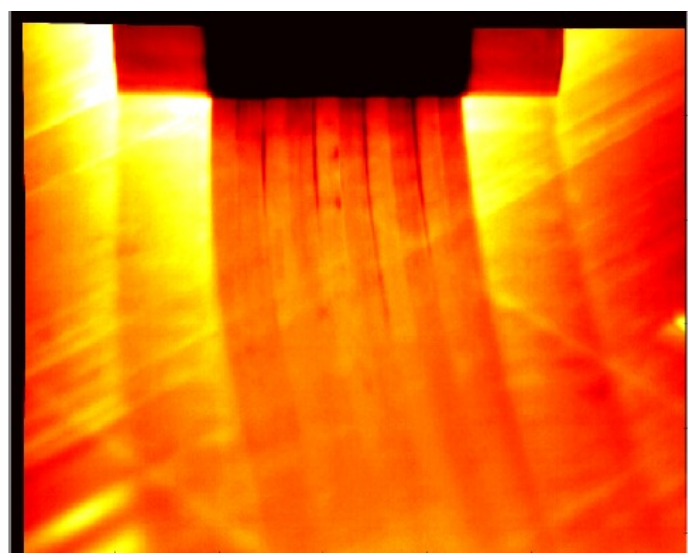

(b)

Figure 12. (a) In situ thermal image of tow-steered composite layup showing some fiber tow peel-up. (b) In situ thermal image of tow-steered composite layup showing low peel-up, good adhesion to the substrate.

\section{CONCLUSIONS}

This paper presented a review of ongoing NDE inspection research and simulation tool development within the NASA's NESB at Langley Research Center. The paper gave an overview of a few of the technologies under development for rapid, large-area inspection of complex composite structures. Examples given show how those technologies are being applied to characterize the state of composite structures. The rapid, quantitative results produced by these technologies provide one step in the process of sustainment for large composite structures during a vehicle's life. Additionally, a discussion of emerging technology areas for NDE were presented, including examples incorporating realistic composite damage, model based data analysis for faster, more reliable results and in-process monitoring of composite part fabrication. A number of other techniques being explored by NESB for characterization of composite structures were not discussed in this paper. For example, current research includes the use of fiber optic, wireless surface acoustic wave and acoustic emission sensors for structural health monitoring (SHM) of large composite components, eddy current inspection of high temperature ceramic composites as well as terahertz and microwave inspection of non-conducting composite materials. Finally, NESB is investigating how data from multiple NDE and/or SHM techniques applied to the same structure can be combined to provide a fuller understanding of the overall health of component under inspection.

\section{ACKNOWLEDGEMENTS}

The author would like to thank the following members of the Nondestructive Evaluation Sciences Branch for their contributions to this paper: Joshua Brown, Elizabeth Gregory, Patricia Howell, Patrick Johnston, Cara Leckey, Jeffery Seebo and Joseph Zalameda.

\section{REFERENCES}

[1] Griffiths, B., "Boeing sets pace for composite usage in large civil aircraft," High Performance Composites, 5(1), (2005).

[2] Kirsch, M. T., "Composite Crew Module: Primary Structure," NASA Technical Memorandum NASA/TM-2011217185, NESC-RP-06-0191, (2011).

[3] Vickers, J. H., et. al., "NASA Composite Cryotank Technology Demonstration: Overview", SAMPE 2012 Conference, Baltimore, MD, (2011).

[4] Canturri, C., et. al., "Delamination growth directionality and the subsequent migration processes-The key to damage tolerant design." Composites Part A: Applied Science and Manufacturing 54: 79-87, (2013).

[5] Roberts, R., Leckey, C.A.C., "Computational modeling of micro-crack induced attenuation in CFRP composites." Review of Progress in Quantitative Nondestructive Evaluation 32: pp 979-986, 2013). 
[6] Yurgartis, S., "Measurement of small angle fiber misalignments in continuous fiber composites." Comp Sci Technol 30(4): 279-293, (1987).

[7] Cramer. K.E., Winfree, W.P., "Fixed Eigenvector Analysis of Thermographic NDE Data," Proc. SPIE Vol. 8013, (2011).

[8] Winfree, W.P., et. al., "Principal Component Analysis of Thermographic Data," Proc. SPIE 9485, (2015).

[9] Gazarik, M., et. al., "Infrared On-orbit RCC Inspection with the EVA IR Camera: Development of Flight Hardware from a COTS System," InfraMation 2005 Proceedings ITC 108A, (2005).

[10] Howell, P.A., et. al., "On-orbit Passive Thermography," Nondestructive Testing and Evaluation, Vol. 23-3: 195210.

[11] Howell, P.A., et. al,, 2007, "DTO-851 Final Report Evaluation of the EVA IR Camera for On-orbit Detection of Subsurface Damage to Reinforced Carbon-carbon,” NASA TP-2007-214895, (2008).

[12] Rotter, H.A., "International Space Station (ISS) Heat Rejection Subsystem (HRS) Radiator Face Sheet Damage," NASA TM-2012-217348, NESC-RP-09-00529, (2012).

[13] Johnston, P. H., et. al., "Ultrasonic monitoring of ply crack and delamination formation in composite tube under torsion load", Ultrasonic Symposium, IEEE: 595-598, (2010).

[14] Johnston, P.H., et. al., "Characterization of delaminations and transverse matrix cracks in composite laminates using multiple-angle ultrasonic inspection," AIP Conference Proceedings Vol. 1511, No. 1: 1011-1018, (2013).

[15] Duran, O., K. et. al., "State of the Art in Sensor Technologies for Sewer Inspection," IEEE Sensors Journal, vol. 2, no. 2:73-81, (2002).

[16] Roman, H.T., "Robotic applications in PSE\&G's nuclear and fossil power plants," IEEE Transactions on Energy Conversion, vol. 8, no. 3: 584-592, (1993).

[17] Pierce, S.G., et. al., "Positioning Challenges in Reconfigurable Semi-autonomous Robotic NDE Inspection," Proc. of SPIE Health Monitoring of Structural and Biological Systems, Vol. 7650: 1C1-1C12, (2010).

[18] Colgate, J. E., et. al., "Cobots: Robots for collaboration with human operators," Proceedings of the 1996 ASME International Mechanical Engineering Congress and Exposition. ASME, (1996).

[19] Leckey, C.A.C., Parker, F.R., "NDE and SHM Simulation for CFRP Composites," Proc. of American Society for Composites 29th Technical Conference, 454, (2014).

[20] Rogge, M., Leckey, C.A.C, "Characterization of impact damage in composite laminates using guided wavefield imaging and local wavenumber domain analysis," Ultrasonics 53: 1217-1226, (2013).

[21] Leckey, C.A.C, et. al., "Guided waves in anisotropic and quasi-isotropic aerospace composites: Three-dimensional simulation and experiment," Ultrasonics 54: 385-394, (2014).

[22] Leckey, C.A.C., Seebo, J.P., "Guided wave energy trapping to detect hidden multilayer delamination damage," Proc. API 1650: 1162-1169, (2015).

[23] Rajic, N., "Principal Component Thermography for Flaw Contrast Enhancement and Flaw Depth Characterisation in Composite Structures," Composite Structures 58: 521-528, (2002).

[24] Rajic, N., "Principal Component Thermography," DSTO-TR-1298, (2002).

[25] Valluzzi, M.R., et. al., "IR thermography for interface analysis of FRP laminates externally bonded to RC beams," Materials and Structures 42: 25-34, (2009).

[26] Genest, M., "Image Processing for Automated Flaw Detection in Pulsed Thermography," Proceeding of 6th International Workshop-NDT Signal Processing: 1-9, (2009).

[27] Vavilov, V. P., Marinetti, S., "Comparative Analysis of the Thermal Testing of Corrosion in High and Low HeatConducting Metals," Russian Journal of Nondestructive Testing 44(12): 841-846, (2008).

[28] Zalameda, J.N., et. al., "Compression Techniques for Improved Algorithm Computational Performance," Proceedings of SPIE, Thermosense XXVII, 5782: 399-406, (2005).

[29] Hermosilla-Lara, S., et. al., "Enhancement of open-cracks detection using a principal component analysis/wavelet technique in photothermal nondestructive testing," Proceedings of Quantitative Infrared Thermography 6: 41-46, (2002).

[30] Marinetti, S. et al., "Statistical analysis of IR thermographic sequences by PCA," Infrared Physics \& Technology 46: 85-91, (2004).

[31] Juarez P.D., et. al., "Advances in In-Situ Inspection of Automated Fiber Placement Systems," SPIE DSS 2016 Sensing Technology Applications, Proc. SPIE Vol. 9861, Thermosense XXXVIII, (2016)

[32] Wilson, W.C., et. al., "October. Carbon fiber TOW angle determination using microwave reflectometry," SENSORS, 2016 IEEE: 1-3, (2016). 